\title{
Phosphodiesterase 10A PET Radioligand Development Program: From Pig to Human
}

\author{
Christophe Plisson ${ }^{1}$, David Weinzimmer ${ }^{2}$, Steen Jakobsen ${ }^{3}$, Sridhar Natesan ${ }^{4}$, Cristian Salinas ${ }^{1}$, Shu-Fei Lin ${ }^{2}$, \\ David Labaree ${ }^{2}$, Ming-Qiang Zheng ${ }^{2}$, Nabeel Nabulsi ${ }^{2}$, Tiago Reis Marques ${ }^{4}$, Shitij Kapur ${ }^{4}$, Eiji Kawanishi ${ }^{5}$, \\ Takeaki Saijo ${ }^{6}$, Roger N. Gunn ${ }^{1}$, Richard E. Carson ${ }^{2}$, and Eugenii A. Rabiner ${ }^{1,5}$ \\ ${ }^{I}$ Imanova Ltd., Centre for Imaging Sciences, Hammersmith Hospital, London, United Kingdom; ${ }^{2}$ Yale University PET Center, \\ Yale University School of Medicine, New Haven, Connecticut; ${ }^{3}$ Aarhus PET Centre, Aarhus Sygehus, Nфrrebrogade, Aarhus \\ C Denmark; ${ }^{4}$ Institute of Psychiatry, King's College, London, United Kingdom; ${ }^{5}$ Medicinal Chemistry Research Laboratories I, \\ Research Division, Mitsubishi Tanabe Pharma Corporation, Yokohama, Japan; and ${ }^{6}$ DMPK Laboratories, Research Division, \\ Mitsubishi Tanabe Pharma Corporation, Chiba, Japan
}

Four novel phosphodiesterase 10A (PDE10A) PET tracers have been synthesized, characterized in preclinical studies, and compared with the previously reported ${ }^{11} \mathrm{C}-\mathrm{MP}-10$. Methods: On the basis of in vitro data, IMA102, IMA104, IMA107, and IMA106 were identified as potential PDE10A radioligand candidates and labeled with either ${ }^{11} \mathrm{C}$ via $\mathrm{N}$-methylation or with ${ }^{18} \mathrm{~F}$ through an $\mathrm{S}_{\mathrm{N}} 2$ reaction, in the case of IMA102. These candidates were compared with ${ }^{11} \mathrm{C}-\mathrm{MP}-10$ in pilot in vivo studies in the pig brain. On the basis of these data, ${ }^{11} \mathrm{C}-\mathrm{IMA} 106$ and ${ }^{11} \mathrm{C}-\mathrm{IMA} 107$ were taken into further evaluation and comparison with ${ }^{11} \mathrm{C}-\mathrm{MP}-10$ in the primate brain. Finally, the most promising radioligand candidate was progressed into human evaluation. Results: All 5 tracers were produced with good radiochemical yield and specific activity. All candidates readily entered the brain and demonstrated a heterogeneous distribution consistent with the known expression of PDE10A. Baseline PET studies in the pig and baboon showed that ${ }^{11} \mathrm{C}-\mathrm{IMA} 107$ and ${ }^{11} \mathrm{C}-\mathrm{MP}-10$ displayed the most favorable tissue kinetics and imaging properties. The administration of selective PDE10A inhibitors reduced the binding of ${ }^{11} \mathrm{C}-$-IMA107 and ${ }^{11} \mathrm{C}-\mathrm{MP}-10$ in the PDE10A-rich brain regions, in a dose-dependent manner. In the nonhuman primate brain, the tissue kinetics of ${ }^{11} \mathrm{C}$ IMA107 and ${ }^{11} \mathrm{C}-\mathrm{MP}-10$ were well described by a 2-tissue-compartment model, allowing robust estimates of the regional total volume of distribution. Blockade with unlabeled MP-10 confirmed the suitability of the cerebellum as a reference tissue and enabled the estimation of regional binding potential as the outcome measure of specific binding. Conclusion: ${ }^{11} \mathrm{C}-\mathrm{IMA} 107$ was identified as the ligand with the highest binding potential while still possessing reversible kinetics. The first human administration of ${ }^{11} \mathrm{C}-\mathrm{IMA} 107$ has demonstrated the expected regional distribution and suitably fast kinetics, indicating that ${ }^{11} \mathrm{C}-$ IMA107 will be a useful tool for the investigation of PDE10A status in the living human brain.

Key Words: PET; PDE10A; ${ }^{11} \mathrm{C}$; in vivo; brain

J Nucl Med 2014; 55:595-601

DOI: 10.2967/jnumed.113.131409

Received Aug. 22, 2013; revision accepted Nov. 4, 2013.

For correspondence or reprints contact: Christophe Plisson, Imanova Ltd., Burlington Danes Bldg., Hammersmith Hospital, Du Cane Rd., London W12 ONN, U.K.

E-mail: christophe.plisson@imanova.co.uk

Published online Mar. 10, 2014.

COPYRIGHT (c) 2014 by the Society of Nuclear Medicine and Molecular Imaging, Inc.
C yclic nucleotide phosphodiesterases (PDEs) are ubiquitous and regulate cellular signal transduction by breaking down cyclic adenosine monophosphate, cyclic guanosine monophosphate, or both $(1,2)$. The PDEs comprise a group of enzymes with several isoforms, and PDE10A, a relatively recently characterized PDE, has a predominant and quite selective brain localization in the basal ganglia $(3,4)$. This spatially limited but high expression suggests that PDE10A may play an important role in basal ganglia neurophysiology. PDE10A is a target for novel drug development aimed at the treatment of central nervous system (CNS) disorders such as schizophrenia (5,6), Huntington disease (7), and Parkinson disease (8). Behavioral studies and postmortem tissue analysis support the involvement of PDE10A in CNS disorders, but direct evidence of its role in these conditions in the human brain is lacking. The ability to quantify PDE10A in the human brain in vivo will aid in understanding its role in neurodegenerative and neuropsychiatric pathophysiology and will provide a powerful tool to enhance the development of novel pharmaceuticals targeting this enzyme.

We previously reported the evaluation of ${ }^{11} \mathrm{C}$-labeled MP-10 in the porcine and primate brain in vivo and demonstrated its utility to quantify PDE10A availability (9); reports of a CNS-penetrant metabolite observed with tracers from MP-10 series $(10,11)$, however, prompted us to investigate radioligand candidates from a different chemical series. Several other PDE10A PET radioligands have recently been described (10-15). ${ }^{18} \mathrm{~F}-J N J 42259152$, an ana$\log$ of MP-10, and ${ }^{18} \mathrm{~F}-\mathrm{MNI} 659$ have been recently reported to be promising candidates for the imaging of PDE10A in humans in vivo $(16,17)$. To our knowledge, no ${ }^{11} \mathrm{C}$-labeled PDE10A radioligands have been reported in human studies. Because of its shorter half-life, ${ }^{11} \mathrm{C}$-labeled ligands offer significant advantages over ${ }^{18} \mathrm{~F}$-labeled ones, both in terms of the reduced radiation exposure to study participants and in the facilitation of multiple scanning sessions in the same individual on the same day. The ability to conduct multiple PET scans in the same day is critical in drug development when performing time-course occupancy studies required to evaluate the relationship between plasma and brain pharmacokinetics. In addition, ${ }^{11} \mathrm{C}$-labeled ligands allow multiple daily syntheses on the same platform, providing optimization of imaging infrastructure.

We demonstrated here the development and translation of a novel PDE10A radioligand. An in vitro and feasibility assessment identified 4 candidates, which were progressed to an initial evaluation 
in the pig brain. The leading 2 molecules were then evaluated more thoroughly in the nonhuman primate. The most favorable candidate, ${ }^{11} \mathrm{C}$-IMA107, was progressed into human testing and used to obtain the first human images.

\section{MATERIALS AND METHODS}

\section{Chemistry}

All the precursor and reference compounds were prepared by Mitsubishi Tanabe Pharma Corp. according to procedures previously reported (18).

\section{In Vitro Measurement of PDE10 Inhibitory Activity}

The human enzyme PDE10A (hPDE10A) was isolated from COS-7 cells transfected with plasmid coding for human PDE10A2, according to the methods described by Kotera et al. (19). The PDE assays were performed according to a modified method reported by Kotera et al. (20). A description of the assay can be found in the supplemental material (available at http://jnm.snmjournals.org).

\section{Radiochemistry}

In vivo pig and baboon experiments were performed at the Aarhus and Yale PET centers, respectively. ${ }^{11} \mathrm{C}-\mathrm{MP}-10$ radiosynthesis at both centers was previously reported (9). The radiosyntheses of ${ }^{11} \mathrm{C}$-IMA104, ${ }^{11} \mathrm{C}$-IMA107, and ${ }^{11} \mathrm{C}$-IMA106 were all performed under similar conditions. The labeling procedures of the ${ }^{11} \mathrm{C}$ - and ${ }^{18} \mathrm{~F}$-pyrazolopyrimidine compounds ${ }^{11} \mathrm{C}$-IMA107 and ${ }^{18} \mathrm{~F}$-IMA102 are reported in the supplemental material.

\section{Pig PET Studies}

All animal studies were performed in accordance with the Danish Animal Experimentation Act on a license granted by the Danish Ministry of Justice. A description of the subjects' preparation and monitoring, PET data acquisition, image processing, and blocking experiments can be found in the supplemental material.

\section{Baboon PET Studies}

The study was approved by the local Institutional Animal Care and Use Committee. A description of the subjects' preparation and monitoring, PET data acquisition, image processing, arterial plasma input function generation, tracer kinetic analysis, and blocking experiments can be found in the supplemental material.

\section{Human Studies}

The data in this manuscript come from the first 2 scans obtained in an ongoing human study at Imanova Ltd. The Research Ethics Committee approved this study, and all subjects signed a written informed consent form. Each subject underwent a PET scan on a Siemens PET/CT TruePoint Biograph 6-camera system, after the administration of ${ }^{11} \mathrm{C}$ IMA107 (147-312 MBq) as a 20-s bolus. The specific activity at the time of injection was $62-287 \mathrm{GBq} / \mu \mathrm{mol}$. Before the PET scan, each subject was cannulated in the forearm or antecubital vein for tracer administration and in the contralateral radial artery for blood sampling. After a low-dose CT transmission scan, a 90-min dynamic emission scan was acquired. Each subject also underwent volumetric MR imaging to aid in the analysis of the PET data.

\section{RESULTS}

\section{In Vitro Affinity Determination}

The selected compounds showed subnanomolar affinity for the hPDE10A, with inhibitory concentration of $50 \%\left(\mathrm{IC}_{50}\right)$ values of $0.02,0.02,0.79$, and $0.06 \mathrm{nM}$ for MP-10, IMA106, IMA102, and IMA107, respectively. An affinity assay of IMA104 was conducted using bovine PDE10A; IMA104 exhibited high affinity, with an $\mathrm{IC}_{50}$ value of $0.01 \mathrm{nM}$. IMA104 was not tested at the
hPDE10A; however, other compounds showed similar affinity in our assay for both the bovine and the human PDE10A. The selectivity of IMA104 was not determined; however, the other compounds showed high selectivity for hPDE10A toward other PDEs, with an $\mathrm{IC}_{50}$ greater than $1,000 \mathrm{nM}$, except for IMA102, which showed a PDE4 $\mathrm{IC}_{50}$ of $61 \mathrm{nM}$. The selectivities of IMA102, IMA107, and MP-10 were also tested in vitro against a variety of CNS receptors and enzymes, and no secondary targets of relevance were seen. For IMA107, the highest-affinity secondary target was $5.8 \mu \mathrm{M}$, with a 100,000-fold-lower affinity than PDE10 (Supplemental Table 1).

\section{Radiochemistry}

All ${ }^{11} \mathrm{C}$ radiotracers were successfully labeled by $N$-alkylation of the secondary amine precursor with ${ }^{11} \mathrm{C}$-methyl iodide (Fig. 1). They could be routinely produced in more than a 2-GBq yield, starting from a typical cyclotron bombardment of $55 \mu \mathrm{A}$ for $40 \mathrm{~min}$, which corresponds to an estimated $30 \%$ decay-corrected radiochemical yield from ${ }^{11} \mathrm{C}$-carbon dioxide. The total synthesis times were about 40 min including purification and formulation counted from the end of bombardment. The radiosynthesis of ${ }^{18} \mathrm{~F}$-IMA102 was achieved by nucleophilic substitution of the mesylate precursor IMA101 with ${ }^{18} \mathrm{~F}$-fluoride with inversion of the configuration (Fig. 1). The radiochemical yield of this reaction was good, with $11 \mathrm{GBq}$ produced from a 60 -min irradiation at $40 \mu \mathrm{A}$, which corresponds to an estimated $22 \%$ non-decay-corrected yield. The radiochemical purity of all tracers was greater than $95 \%$, as determined by analytic high-performance liquid chromatography with radiodetection. No traces of precursor were detected in the injected doses of all of the radiotracers examined, and only tracer level amount of unknown chemical species was observed. The specific activity of

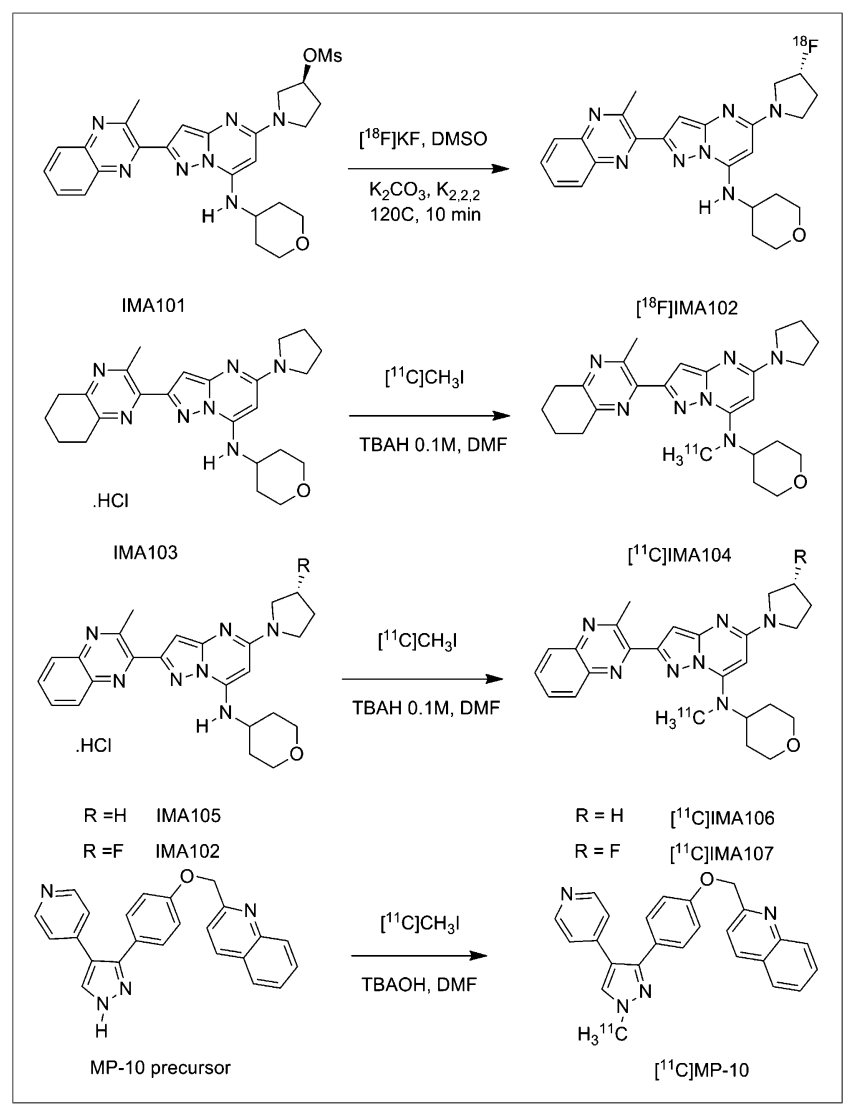

FIGURE 1. Radiosynthesis of PDE10A radioligand candidates. 


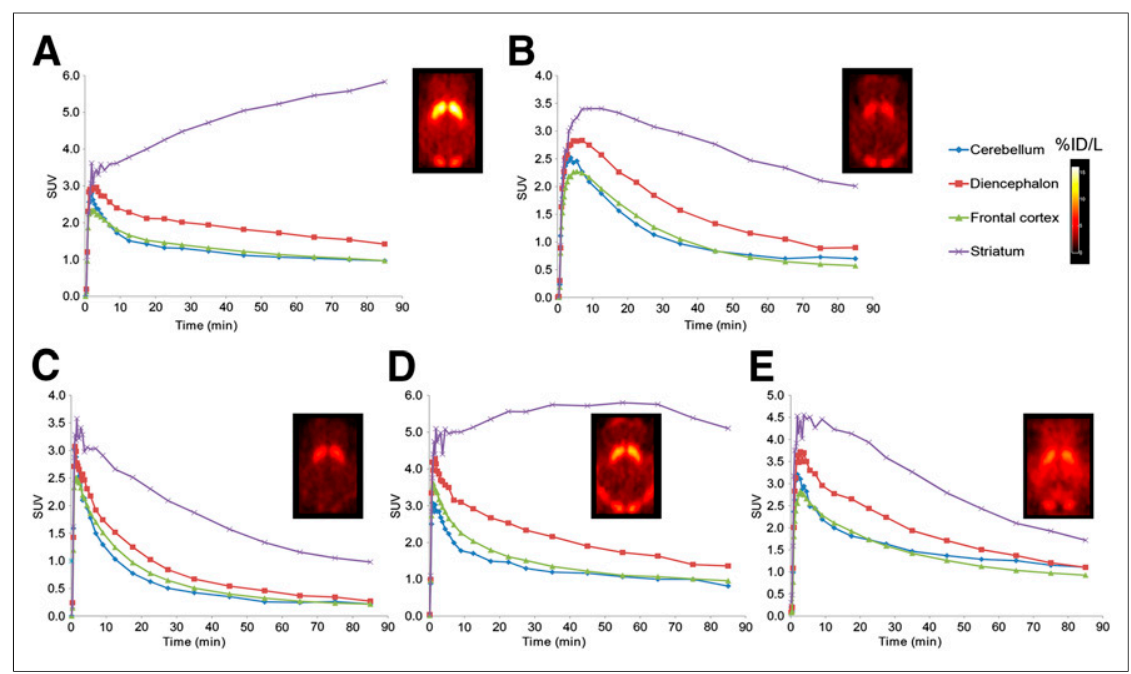

FIGURE 2. Summed PET images in pig brain and representative time-activity curves for ${ }^{11} \mathrm{C}$ IMA104 (A), ${ }^{11} \mathrm{C}$-IMA107 (B), ${ }^{11} \mathrm{C}-\mathrm{MP}-10$ (C), ${ }^{11} \mathrm{C}-$ IMA106 (D), and ${ }^{18} \mathrm{~F}-$-IMA102 (E) for selected ROIs. Images displayed at level of striatum in transverse orientations are expressed as percentage injected dose per liter (\%ID/L). SUVs are tissue radioactivity concentrations normalized for radioactive dose injected and for subject's body mass.

${ }^{11} \mathrm{C}$-MP-10, ${ }^{11} \mathrm{C}$-IMA106, ${ }^{11} \mathrm{C}$-IMA104, ${ }^{11} \mathrm{C}$-IMA107, and ${ }^{18} \mathrm{~F}$-IMA102 at the end of synthesis was $70 \pm 92(n=7), 214(n=1), 87(n=1)$, $121 \pm 47(n=3)$, and $250 \mathrm{GBq} / \mu \mathrm{mol}(n=1)$, respectively, for the pig study. The specific activity of ${ }^{11} \mathrm{C}-\mathrm{MP}-10,{ }^{11} \mathrm{C}-\mathrm{IMA} 106$, and ${ }^{11} \mathrm{C}$-IMA107 at the end of synthesis was $285 \pm 207(n=16)$, $19(n=1)$, and $68 \pm 24 \mathrm{GBq} / \mu \mathrm{mol}(n=4)$, respectively, for the primate study.

\section{Pig PET Studies}

All the radiotracers readily entered the brain and showed a heterogeneous CNS distribution in the pig. PET images of summed radioactivity from 0 to $90 \mathrm{~min}$, showing the regional distribution of the tracers at baseline, are depicted in Figure 2. The brain region demonstrating the highest accumulation and retention of radioactivity was the striatum, consistent with reported qualitative immunohistochemical staining indicating the highest expression of PDE10A in this region $(4,21)$ and consistent with previous experience with other PDE10A radioligands. The highest striatum-tosurrounding brain contrast was seen for ${ }^{11} \mathrm{C}$-IMA104. The regional tissue time-activity curves for each radiotracer are depicted in Figure 3. For all tracers, the highest uptake was seen in the striatum, followed by the diencephalon, with lower uptake in the frontal

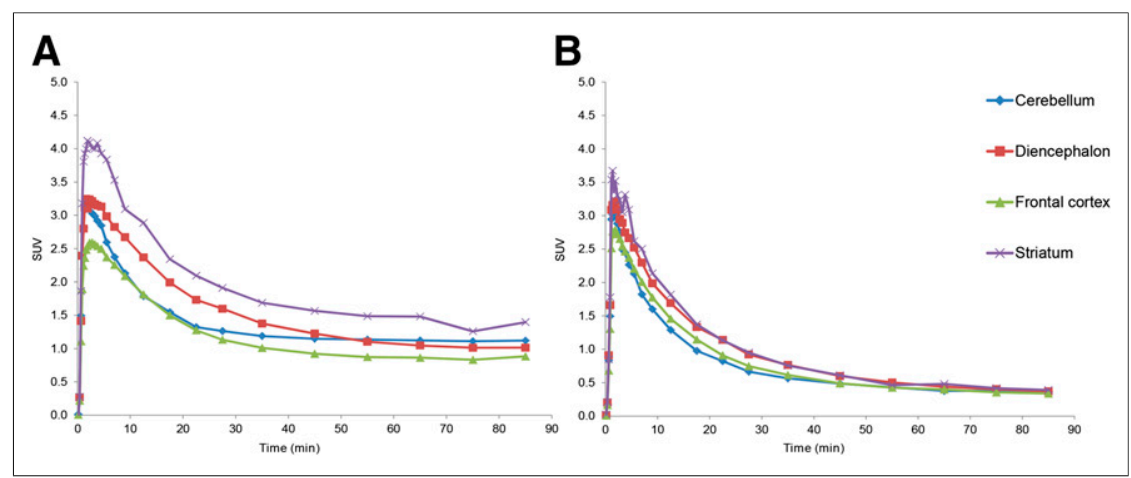

FIGURE 3. Time-activity curves describing kinetics of ${ }^{11} \mathrm{C}-\mathrm{IMA} 107$ (A) and ${ }^{11} \mathrm{C}-\mathrm{MP}-10$ (B) for selected ROls in pig brain after administration of IMA102 doses of $0.2 \mathrm{mg} / \mathrm{kg}(\mathrm{A})$ and $5 \mathrm{mg} / \mathrm{kg}(\mathrm{B})$. cortex and cerebellum, which showed similar levels of uptake. ${ }^{11} \mathrm{C}$-IMA107, ${ }^{11} \mathrm{C}-\mathrm{MP}$ 10, and ${ }^{18} \mathrm{~F}$-IMA102 exhibited a fast washout from all regions of interest (ROIs), whereas ${ }^{11}$ C-IMA104 and ${ }^{11}$ C-IMA106 showed slower kinetics in the PDE10A-rich striatum. ${ }^{11} \mathrm{C}$ IMA106 uptake in the striatum peaked by $35 \mathrm{~min}$ and plateaued between 40 and $60 \mathrm{~min}$, displaying a slight decrease from then on. For ${ }^{11} \mathrm{C}$-IMA104, the striatal signal continued increasing during the 90-min scanning session.

Blocking studies of ${ }^{11} \mathrm{C}-\mathrm{MP}-10$ and ${ }^{11} \mathrm{C}$ IMA107 were performed using a pharmacologic dose of IMA102 (Fig. 3, time-activity curves). After the administration of the blocker, the uptake of both tracers in the striatum was significantly reduced, reaching the levels of other CNS tissues at the highest dose of the blocker $(5 \mathrm{mg} / \mathrm{kg})$.

\section{Baboon PET Studies}

Representative baseline standardized uptake images for ${ }^{11} \mathrm{C}-\mathrm{MP}-10,{ }^{11} \mathrm{C}$-IMA107, and ${ }^{11} \mathrm{C}$-IMA106 in the baboon brain are given in Figure 4 . All 3 tracers readily entered the baboon brain and showed a regional uptake consistent with our findings in the pig. The time-activity curves depicted in Figure 4 revealed that the rank order of uptake was similar for all tracers, with the putamen $>$ caudate $=$ globus pallidus $>$ nucleus accumbens $>$ remaining ROIs. All 3 tracers displayed a comparable initial uptake in the ROIs, in which the PDE10A protein is expressed at a low level, with a standardized uptake value (SUV) around 1.5 at $5 \mathrm{~min}$ after injection. ${ }^{11} \mathrm{C}-\mathrm{MP}$ 10 and ${ }^{11} \mathrm{C}$-IMA107 uptake reached peak levels by 50 and $35 \mathrm{~min}$ after administration, respectively, and declined after that. In contrast, ${ }^{11} \mathrm{C}$-IMA106 tissue kinetics were slower, with no decrease observed in the PDE10A-rich regions during the 120-min scanning time.

The in vivo metabolism of all tracers was determined in all scanning sessions and showed that the 3 tracers were metabolized, albeit, to somewhat different extents (Supplemental Fig. 1). The rate of metabolism of the pyrazolopyrimidine tracers, ${ }^{11} \mathrm{C}$-IMA107 ( $\sim 50 \%$ of parent at $90 \mathrm{~min}$ ) and ${ }^{11} \mathrm{C}-\mathrm{IMA} 106(56 \%$ of parent at $90 \mathrm{~min})$, were slower than that of ${ }^{11} \mathrm{C}-\mathrm{MP}-10(\sim 30 \%$ of parent at $90 \mathrm{~min})$. All the metabolites observed were more polar than their parent compound.

The 2-tissue-compartment analysis with metabolite-corrected plasma input was able to accurately characterize the kinetic behavior of the 3 tracers (time-activity curves and model fits for ${ }^{11} \mathrm{C}$-IMA107 can be found in Supplemental Fig. 2). The volume of distribution $\left(V_{\mathrm{T}}\right)$ for all regions considered and all 3 tracers are presented in Table 1. The $V_{\mathrm{T}}$ values at baseline for all tracers were highest in the putamen and lowest in all ROIs outside the basal ganglia. ${ }^{11} \mathrm{C}$-IMA107 and ${ }^{11} \mathrm{C}$-IMA106 with rate constant for transfer from arterial plasma to tissue $\left(K_{1}\right)$ values at baseline of $0.38 \pm$ 0.13 and 0.413 , respectively, showed higher delivery than ${ }^{11} \mathrm{C}-\mathrm{MP}-10(0.138 \pm 0.05)$. 


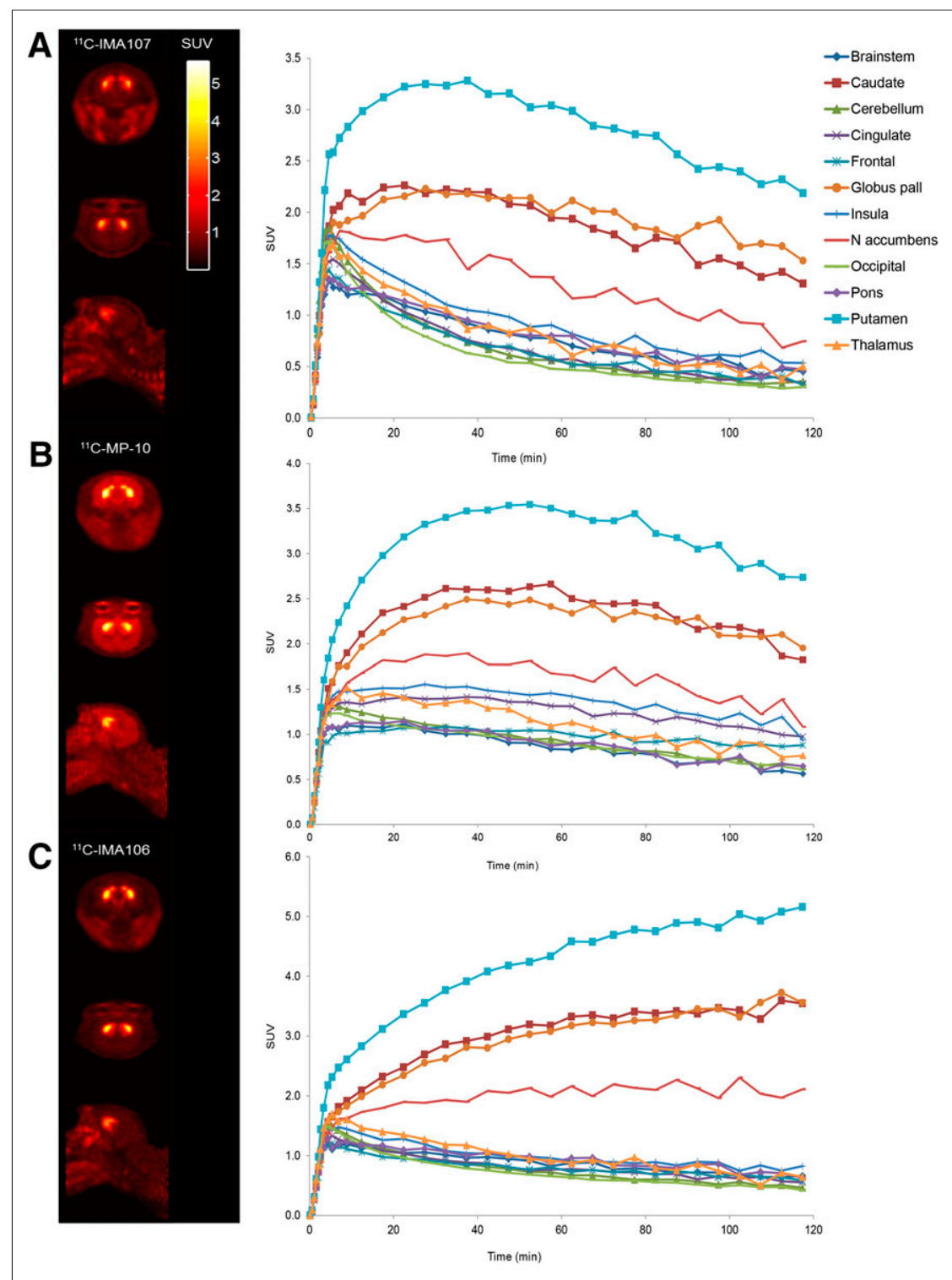

FIGURE 4. PET images in baboon brain summed from 60 to 90 min and baseline time-activity curves after bolus injection of ${ }^{11} \mathrm{C}-\mathrm{IMA} 107$ (A), ${ }^{11} \mathrm{C}-\mathrm{MP}-10$ (B), and ${ }^{11} \mathrm{C}-\mathrm{IMA} 106$ (C). Image slices are displayed in SUV units at level of striatum in coronal, transverse, and sagittal orientations. studies with unlabeled MP-10 demonstrated that the tracers' binding is specific to binding to the PDE10A enzyme. The $V_{\mathrm{T}}$ of the cerebellum did not decrease significantly after treatment and confirmed the suitability of the cerebellum as a reference region and allowed for the regional estimation of $B P_{\mathrm{ND}} B P_{\mathrm{ND}}$ estimates obtained under baseline conditions are given in Table 2 . ${ }^{11} \mathrm{C}$-IMA106 slow tissue kinetics yielded the highest $B P_{\mathrm{ND}}$ estimates in the PDE10A-rich regions with, for example, a value of 12.71 in the putamen.

The occupancy levels obtained after administration of unlabeled MP-10 were consistent using either radioligand, with occupancy values at the dose of 0.6 and $1.8 \mathrm{mg} / \mathrm{kg}$ of $70 \%$ and $85 \%$ for ${ }^{11} \mathrm{C}-\mathrm{MP}-10$ and $63 \%$ and $85 \%$ for ${ }^{11} \mathrm{C}$-IMA 107 .

\section{Human Studies}

There were no adverse or clinically detectable pharmacologic effects in any of the subjects. No significant changes in vital signs or the results of laboratory studies or electrocardiograms were observed after administration of ${ }^{11} \mathrm{C}$-IMA107. Representative images from a baseline scan are shown in Figure 6. The regional distribution images of ${ }^{11} \mathrm{C}$-IMA107 in the human brain were similar to those of the pig and monkey brain. After intravenous administration of ${ }^{11} \mathrm{C}$-IMA107, radioactivity readily entered the brain, with an SUV of up to 3 in the putamen at peak uptake. The distribution was heterogeneous, with highest concentrations in the putamen and globus pallidus. The time-activity curves from a baseline scan are shown in Figure 6. The kinetics of the uptake of ${ }^{11} \mathrm{C}$-IMA107 in the brain were reversible, with a measurable washout of the ligand within the time frame of the PET scans, seen in the regional timeactivity curves.
The slower kinetics of ${ }^{11} \mathrm{C}$-IMA106 were reflected in the higher $V_{\mathrm{T}}$ values for this tracer.

The magnitude of the specific binding component of ${ }^{11} \mathrm{C}$ IMA107 and ${ }^{11} \mathrm{C}-\mathrm{MP}-10$ was assessed after blocking experiments with unlabeled MP-10 at doses of 0.6 and $1.8 \mathrm{mg} / \mathrm{kg}$. A dosedependent decrease in tracer uptake was observed in the PDE10A-rich regions; the time-activity curves obtained for ${ }^{11} \mathrm{C}$ IMA107 and ${ }^{11} \mathrm{C}-\mathrm{MP}-10$ after administration of the highest dose of MP-10 (1.8 mg/kg) are depicted in Figure 5 and showed a reduction of the tracer uptake in the striatal regions to a level close to that of the cerebellum, as previously reported for ${ }^{11} \mathrm{C}-\mathrm{MP}-10$ (9). The $K_{1}$ values for ${ }^{11} \mathrm{C}$-IMA107 at baseline $(0.38 \pm 0.13)$ and after block $(0.428 \pm 0.09)$ were consistent, reflecting an unchanged tracer delivery. The quantification of the ${ }^{11} \mathrm{C}-\mathrm{MP}-10$ and ${ }^{11} \mathrm{C}$ IMA107 binding confirmed that the administration of MP-10 induces a profound decrease in the regional $V_{\mathrm{T}}$ in the striatum and globus pallidus for both tracers (Table 1). These in vivo blocking

\section{DISCUSSION}

The aim of this study was to develop a PET radioligand for imaging PDE10A in the human brain in vivo. We previously reported the labeling and evaluation of ${ }^{11} \mathrm{C}-\mathrm{MP}-10$; however, in parallel to the ${ }^{11} \mathrm{C}-\mathrm{MP}-10$ evaluation, we also investigated the characteristics of pyrazolopyrimidine PDE10A inhibitors. The 4 pyrazolopyrimidine compounds (as well as ${ }^{11} \mathrm{C}-\mathrm{MP}-10$ ) were successfully labeled using ${ }^{11} \mathrm{C}$-methyl iodide or ${ }^{18} \mathrm{~F}$-fluoride and produced with high yield, specific activity, and radiochemical purity. Although the radiosynthesis of ${ }^{11} \mathrm{C}-\mathrm{MP}-10$ leads to a regioisomer, the radiochemical yields of the ${ }^{11} \mathrm{C}$-pyrazolopyrimidine were only slightly better than those obtained for ${ }^{11} \mathrm{C}-\mathrm{MP}-10$. Analysis of the semipreparative high-performance liquid chromatograms showed that the percentage of product in the crude reaction was around $60 \%$ for ${ }^{11} \mathrm{C}$-IMA107, compared with $49 \%$ for ${ }^{11} \mathrm{C}-\mathrm{MP}-10$. Labeling of IMA107 with ${ }^{18} \mathrm{~F}$ has not been attempted, however, it is 
TABLE 1

Estimates of $V_{T}$ for Baboon Brain ROls Before and After Administration of MP-10

\begin{tabular}{|c|c|c|c|c|c|}
\hline \multirow[b]{2}{*}{$\mathrm{ROI}$} & \multicolumn{2}{|c|}{${ }^{11} \mathrm{C}-\mathrm{MP}-10$} & \multicolumn{2}{|c|}{${ }^{11} \mathrm{C}-I M A 107$} & \multirow[b]{2}{*}{${ }^{11} \mathrm{C}-\mathrm{IMA} 106$, baseline } \\
\hline & Baseline & $1.8 \mathrm{mg} / \mathrm{kg}$ & Baseline & $1.8 \mathrm{mg} / \mathrm{kg}$ & \\
\hline Putamen & $9.62 \pm 1.75$ & 3.39 & $22.48 \pm 0.15$ & 7.34 & 68.13 \\
\hline Caudate & $7.03 \pm 0.82$ & 2.85 & $13.94 \pm 0.24$ & 5.70 & 41.03 \\
\hline Globus pallidus & $6.38 \pm 1.21$ & 3.47 & $16.66 \pm 0.46$ & 8.52 & 45.02 \\
\hline Nucleus accumbens & $4.33 \pm 0.92$ & 2.57 & $8.49 \pm 0.14$ & 4.45 & 20.00 \\
\hline Insula & $3.84 \pm 0.38$ & 2.47 & $5.71 \pm 0.03$ & 4.56 & 6.65 \\
\hline Cingulate & $3.46 \pm 0.39$ & 2.45 & $4.31 \pm 0.49$ & 4.10 & 5.39 \\
\hline Frontal cortex & $3.14 \pm 0.30$ & 2.27 & $4.10 \pm 0.26$ & 3.84 & 5.66 \\
\hline Thalamus & $3.04 \pm 0.32$ & 2.48 & $5.42 \pm 0.78$ & 4.85 & 6.55 \\
\hline Occipital & $2.39 \pm 0.18$ & 2.03 & $3.83 \pm 0.48$ & 3.58 & 4.64 \\
\hline Pons & $2.10 \pm 0.16$ & 2.50 & $5.66 \pm 1.22$ & 5.69 & 6.48 \\
\hline Brain stem & $2.03 \pm 0.09$ & 2.43 & $5.42 \pm 1.13$ & 5.41 & 5.95 \\
\hline Cerebellum & $2.46 \pm 0.16$ & 2.38 & $4.33 \pm 0.72$ & 4.17 & 4.97 \\
\hline
\end{tabular}

Baseline values are mean and SD of 12 and 2 scans for ${ }^{11} \mathrm{C}-\mathrm{MP}-10$ and ${ }^{11} \mathrm{C}-\mathrm{IMA} 107$, respectively.

most likely that this could be achieved using the same labeling route as for IMA102. Although the shorter half-life of ${ }^{11} \mathrm{C}$ is preferred when multiple scans in the same subject are performed on the same day, the ${ }^{18} \mathrm{~F}$-labeled analog could be attractive to centers with no on-site cyclotron or for distribution.

All the radiotracers were initially evaluated in the pig brain in view of selecting the best candidates for further investigation in the nonhuman primate. The uptake for all candidates was heterogeneous, with high uptake localized to the striatum, consistent with the known expression of the enzyme. The tracers' kinetics and striatum-to-cerebellum ratio at $90 \mathrm{~min}$ seen in vivo correlated well with the in vitro affinity. ${ }^{11} \mathrm{C}$-IMA107, ${ }^{11} \mathrm{C}-\mathrm{MP}-10$, and ${ }^{11} \mathrm{C}$ IMA106 exhibited suitably fast kinetics in the pig brain. The other candidates were less promising, with a low striatum-to-rest-ofbrain ratio in the case of ${ }^{18} \mathrm{~F}$-IMA102 and slow kinetics that did not appear favorable for quantification in the case of ${ }^{11} \mathrm{C}$-IMA104. In vivo blocking studies with unlabeled IMA102 demonstrated that the uptake of ${ }^{11} \mathrm{C}$-IMA107 and ${ }^{11} \mathrm{C}-\mathrm{MP}-10$ in the striatum was significantly reduced after administration of a PDE10A inhibitor, suggesting that both the pyrazolopyrimidine compound series and the MP-10 demonstrate specific binding for PDE10A.

On the basis of these results, ${ }^{11} \mathrm{C}-\mathrm{MP}-10,{ }^{11} \mathrm{C}$-IMA107, and ${ }^{11} \mathrm{C}$ IMA106 were selected for in vivo evaluation in higher species. In the baboon brain, all 3 tracers displayed slower brain kinetics; this was particularly noticeable for ${ }^{11} \mathrm{C}$-IMA106 (Fig. 4C). Although

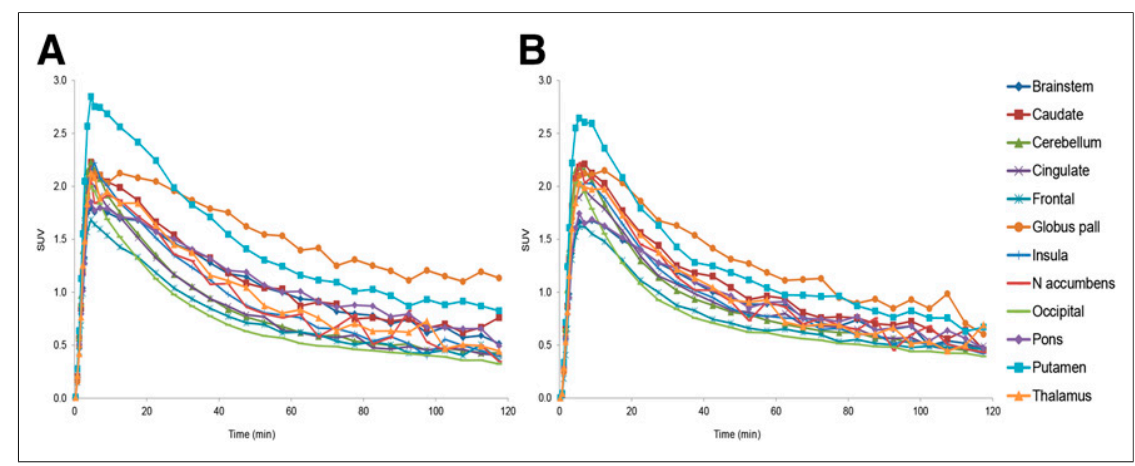

FIGURE 5. Time-activity curves for selected ROls in baboon brain after bolus injection of ${ }^{11} \mathrm{C}-\mathrm{IMA} 107$ (A) and ${ }^{11} \mathrm{C}-\mathrm{MP}-10$ (B) after administration of MP-10 (1.8 mg/kg). quantification was possible in the baboon with this tracer, the slower kinetics suggested that it was not an ideal candidate for translation to humans. As a result, the binding selectivity of ${ }^{11} \mathrm{C}$-IMA106 was not explored. However, the higher affinity of ${ }^{11} \mathrm{C}$-IMA106 that led to a higher signal-to-noise ratio may facilitate imaging of extrastriatal regions such as the substantia nigra, in which PDE10A expression is known to be significantly lower. On the other hand, ${ }^{11} \mathrm{C}$-IMA107 and ${ }^{11} \mathrm{C}-\mathrm{MP}-10$ demonstrated good brain penetration, reversible kinetics, and a good specific signal that could be reduced in a dose-dependent manner after competition with unlabeled MP-10. It is worth mentioning that the kinetics of both tracers in the globus pallidus appeared different from those of other basal ganglia regions, displaying a slower washout. We do not believe this phenomenon can be explained by the tracers or a metabolite binding to a different target in this region, because this effect was observed with both tracers, under both baseline and homologous competition conditions.

Quantitative analysis of ${ }^{11} \mathrm{C}-\mathrm{MP}-10$ and ${ }^{11} \mathrm{C}$-IMA107 binding in the baboon brain determined that both ligands possessed good in vivo radioligand characteristics for imaging PDE10A. Analyses of data before and after competition with a PDE10A inhibitor confirmed the specificity of the ${ }^{11} \mathrm{C}$-IMA107 and ${ }^{11} \mathrm{C}$-MP-10 binding and the suitability of the cerebellum as a reference region for the determination of the regional estimation of $B P_{\mathrm{ND}}$. As previously indicated for ${ }^{11} \mathrm{C}-\mathrm{MP}-10$ (9), this implies that the quantitative analysis of PDE10A PET ligands may not necessitate the accurate measurement of plasma radioactivity and metabolites. The occupancy levels in the striatum calculated for the pharmacologic doses of MP-10 were similar for both radioligands. The high occupancy of $85 \%$ measured at the $1.8 \mathrm{mg} / \mathrm{kg}$ dose confirmed the specificity of the radioligand binding in this region and indicated that changes in the PDE10A availability in the striatum can be quantitatively measured using either radioligand. The globus pallidus was, however, excluded from the occupancy determination because the occupancy values in this region were similar but lower for the 2 tracers at $68 \%$ and $69 \%$, confirming the 
TABLE 2

Baseline $B P_{\mathrm{ND}}$ in Selected Baboon Brain Regions for ${ }^{11} \mathrm{C}-\mathrm{MP}-10(n=12),{ }^{11} \mathrm{C}-\mathrm{IMA} 107(n=2)$, and ${ }^{11} \mathrm{C}-\mathrm{IMA} 106(n=1)$

\begin{tabular}{|c|c|c|c|c|c|}
\hline \multirow[b]{2}{*}{$\mathrm{ROI}$} & \multicolumn{2}{|c|}{${ }^{11} \mathrm{C}-\mathrm{MP}-10$} & \multicolumn{2}{|c|}{${ }^{11} \mathrm{C}-\mathrm{IMA} 107$} & \multirow[b]{2}{*}{${ }^{11} \mathrm{C}-\mathrm{IMA} 106$, mean } \\
\hline & Mean & SD & Mean & SD & \\
\hline Putamen & 2.93 & 0.71 & 4.26 & 0.84 & 12.71 \\
\hline Caudate & 1.86 & 0.35 & 2.26 & 0.49 & 7.26 \\
\hline Globus pallidus & 1.62 & 0.49 & 2.89 & 0.54 & 8.06 \\
\hline Nucleus accumbens & 0.77 & 0.38 & 0.99 & 0.36 & 3.02 \\
\hline Insula & 0.57 & 0.15 & 0.34 & 0.23 & 0.34 \\
\hline Cingulate & 0.41 & 0.16 & 0.00 & 0.05 & 0.08 \\
\hline Frontal cortex & 0.27 & 0.12 & -0.04 & 0.10 & 0.14 \\
\hline Thalamus & 0.23 & 0.09 & 0.25 & 0.03 & 0.32 \\
\hline Occipital & -0.03 & 0.07 & -0.11 & 0.04 & -0.07 \\
\hline Pons & -0.14 & 0.05 & 0.30 & 0.06 & 0.30 \\
\hline Brain stem & -0.17 & 0.04 & 0.25 & 0.05 & 0.20 \\
\hline
\end{tabular}

differential behavior of both tracers in this region, compared with the striatum. We cannot exclude regional differences between the nonspecific binding in the globus pallidus and striatal regions.

Qualitative assessment of ${ }^{11} \mathrm{C}-\mathrm{MP}-10$ uptake in the regions of low or negligible PDE10A expression indicated that the distribution of ${ }^{11} \mathrm{C}-\mathrm{MP}-10$ appears slightly different from that of the pyrazolopyrimidine tracers, with a higher relative uptake in the insula and cingulate (Fig. 4). The quantitative analysis confirmed the higher uptake of ${ }^{11} \mathrm{C}-\mathrm{MP}-10$ in these regions, with $B P_{\mathrm{ND}}$ values of 0.41 and 0.57 in the cingulate and insula, respectively, compared with those of the pyrazolopyrimidine tracers, with $B P_{\mathrm{ND}}$ values in the cingulate and insula of 0 and 0.34 for ${ }^{11} \mathrm{C}$-IMA107 and 0.08 and 0.34 for ${ }^{11} \mathrm{C}$-IMA106, respectively. The uptake of ${ }^{11} \mathrm{C}-\mathrm{MP}-10$ in these regions was also blocked after administration of MP-10 (1.8 $\mathrm{mg} / \mathrm{kg}$ ), with $B P_{\mathrm{ND}}$ values in the cingulate and insula reduced to 0.03 and 0.04 , respectively ( $P$ values calculated using a 1 -tail $t$ test $\leq 0.02$ ). The magnitude of the signal change in these regions was similar to that observed in the putamen and caudate. However, blocking experiments performed with a structurally different PDE10A inhibitor (data not shown) showed that a dose producing over $95 \%$ occupancy in the striatal regions did not significantly reduce the ${ }^{11} \mathrm{C}-\mathrm{MP}-10 B P_{\mathrm{ND}}$ values in the insula and cingulate ( $P$ values calculated using a 1 -tail $t$ test $\geq 0.69$ ). The ${ }^{11} \mathrm{C}-\mathrm{MP}-10$ signal reduction in these regions only under homologous pharmacologic challenge may indicate a higher specificity of ${ }^{11} \mathrm{C}$-IMA107, which did not show any reduction of signal in the cingulate and insula after treatment with MP-10. In addition, an ${ }^{11} \mathrm{C}$-IMA107

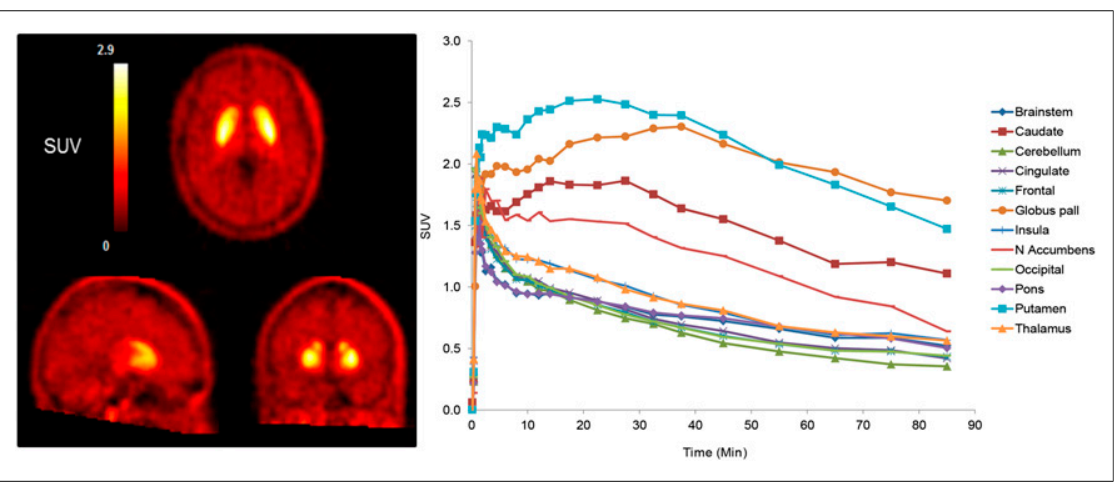

FIGURE 6. Summed PET images in human brain and representative baseline tissue time-activity curves for ${ }^{11} \mathrm{C}$-IMA107 for selected ROIs. Slices are displayed as SUV units at level of striatum in transverse, sagittal, and coronal orientations. effective binding potential, $B P_{\mathrm{ND}}$, of $4.26 \pm 0.84$ in the putamen provides an excellent signal-to-noise ratio, which was superior to that of ${ }^{11} \mathrm{C}-\mathrm{MP}-10$. For these reasons, it was decided to progress ${ }^{11} \mathrm{C}$-IMA107 to humans. After intravenous administration of ${ }^{11} \mathrm{C}$ IMA107 in humans, the regional distribution of radioactivity in the brain was consistent with the distribution previously observed in the pig and baboons. The tracer kinetics were also reversible, indicating a good translation of the tracer behavior between all species studied. Work is in progress to fully characterize ${ }^{11} \mathrm{C}$ IMA107 in humans. Early indications are that it has good signal to noise, with suitably fast kinetics and consistent good kinetics model fits and can be quantified using a simplified reference tissue model.

\section{CONCLUSION}

Four new radioligand candidates for the PDE10A were successfully labeled and compared in vivo in the pig and baboon brains with the previously reported radioligand ${ }^{11} \mathrm{C}-\mathrm{MP}-10 .{ }^{11} \mathrm{C}$-IMA107 proved to be the most suitable for imaging and quantifying PDE10A in preclinical studies. Its initial assessment in humans provides evidence that ${ }^{11} \mathrm{C}$-IMA107 is a promising radioligand for probing the PDE10A in vivo by PET.

\section{DISCLOSURE}

The costs of publication of this article were defrayed in part by the payment of page charges. Therefore, and solely to indicate this fact, this article is hereby marked "advertisement" in accordance with 18 USC section 1734. Part of this research project was performed at the GSK Clinical Imaging Centre before transfer of management to Imanova Ltd. This work was also supported by Mitsubishi Tanabe Pharma Corporation, and the human PET imaging in this project was supported by the National Institute for Health Research (NIHR) Biomedical Research Centre for Mental Health at South London and Maudsley NHS Foundation Trust and the Institute of Psychiatry, King's College London. This article presents independent research and the views expressed are those of the authors and not necessarily 
those of the NHS, the NIHR, or the Department of Health. No other potential conflict of interest relevant to this article was reported.

\section{REFERENCES}

1. Chappie T, Humphrey J, Menniti F, Schmidt C. PDE10A inhibitors: an assessment of the current CNS drug discovery landscape. Curr Opin Drug Discov Devel. 2009;12:458-467.

2. Girault J-A, Greengard P. The neurobiology of dopamine signaling. Arch Neurol. 2004;61:641-644.

3. Soderling SH, Bayuga SJ, Beavo JA. Isolation and characterization of a dualsubstrate phosphodiesterase gene family: PDE10A. Proc Natl Acad Sci USA. 1999;96:7071-7076.

4. Coskran TM, Morton D, Menniti FS, et al. Immunohistochemical localization of phosphodiesterase 10A in multiple mammalian species. J Histochem Cytochem. 2006;54:1205-1213.

5. Siuciak JA, Strick CA. Phosphodiesterase 10A inhibitors as a novel therapeutic approach for schizophrenia. Expert Opin Drug Discov. 2007;2:1001-1009.

6. Schmidt CJ, Chapin DS, Cianfrogna J, et al. Preclinical characterization of selective phosphodiesterase $10 \mathrm{~A}$ inhibitors: a new therapeutic approach to the treatment of schizophrenia. J Pharmacol Exp Ther. 2008;325:681-690.

7. Kleiman RJ, Kimmel LH, Bove SE, et al. Chronic suppression of phosphodiesterase $10 \mathrm{~A}$ alters striatal expression of genes responsible for neurotransmitter synthesis, neurotransmission, and signaling pathways implicated in Huntington's disease. J Pharmacol Exp Ther. 2011;336:64-76.

8. Giorgi M, Melchiorri G, Nuccetelli V, et al. PDE10A and PDE10A-dependent cAMP catabolism are dysregulated oppositely in striatum and nucleus accumbens after lesion of midbrain dopamine neurons in rat: a key step in parkinsonism physiopathology. Neurobiol Dis. 2011;43:293-303.

9. Plisson C, Salinas C, Weinzimmer D, et al. Radiosynthesis and in vivo evaluation of $\left[{ }^{11} \mathrm{C}\right] \mathrm{MP}-10$ as a positron emission tomography radioligand for phosphodiesterase 10A. Nucl Med Biol. 2011;38:875-884.

10. Tu Z, Fan J, Li S, et al. Radiosynthesis and in vivo evaluation of $\left[{ }^{11} \mathrm{C}\right] \mathrm{MP}-10$ as a PET probe for imaging PDE10A in rodent and non-human primate brain. Bioorg Med Chem. 2011;19:1666-1673.
11. Celen S, Koole M, De Angelis M, et al. Preclinical evaluation of ${ }^{18}$ F-JNJ41510417 as a radioligand for PET imaging of phosphodiesterase-10A in the brain. $\mathrm{J} \mathrm{Nucl}$ Med. 2010;51:1584-1591.

12. Andrés J-I, De Angelis M, Alcazar J, et al. Synthesis, in vivo occupancy, and radiolabeling of potent phosphodiesterase subtype-10 inhibitors as candidates for positron emission tomography imaging. J Med Chem. 2011;54: 5820-5835.

13. Hostetler E, Cox CD, Fan H, inventors; Merck Sharp \& Dohme Corp., applicant. Radiolabelled pde10 inhibitors. WO2010/138577A1. Dec. 2, 2010.

14. Funke U, Deuther-Conrad W, Schwan G, et al. Radiosynthesis and radiotracer

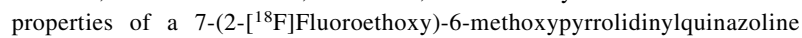
for imaging of phosphodiesterase 10A with PET. Pharmaceuticals. 2012;5: $169-188$.

15. Kehler J, Bang-Andersen B, inventors; H. Lundbeck A/S, applicant. Radiolabelled phenylimidazole-based PDE10A ligands for diagnostic imaging and quantifying PDE10A using PET. WO 2012/062319. Sep. 18, 2013.

16. Van Laere K, Ahmad RU, Hudyana H, et al. Quantification of ${ }^{18}$ F-JNJ-42259152, a novel phosphodiesterase 10A PET tracer: kinetic modeling and test-retest study in human brain. J Nucl Med. 2013;54:1285-1293.

17. Barret $\mathrm{O}$, Thomae $\mathrm{D}$, Alagille $\mathrm{D}$, et al. First in vivo assessment of two PDE10 tracers $\left[{ }^{18} \mathrm{~F}\right] \mathrm{MNI} 654$ and $\left[{ }^{18} \mathrm{~F}\right] \mathrm{MNI} 659$ [abstract]. J Nucl Med. 2012;53(suppl):110P.

18. Kawanishi E, Hongu M, Tanaka Y, inventors; Eiji Kawanishi, Mitsuya Hongu, Yoshihito Tanaka, original assignee. Pyrazolopyrimidine compounds and their use as PDE10 inhibitors. WO2011/105628. Dec. 6, 2012.

19. Kotera J, Fujishige K, Yuasa K, Omori K. Characterization and phosphorylation of PDE10A2, a novel alternative splice variant of human phosphodiesterase that hydrolyzes cAMP and cGMP. Biochem Biophys Res Commun. 1999;261: 551-557.

20. Kotera J, Fujishige K, Michibata H, et al. Characterization and effects of methyl2-(4-aminophenyl)-1,2-dihydro-1-oxo-7-(2-pyridinylmethoxy)-4-(3,4,5-trimethoxyphenyl)-3-isoquinoline carboxylate sulfate (T-1032), a novel potent inhibitor of cGMP-binding cGMP-specific phosphodiesterase (PDE5). Biochem Pharmacol. 2000;60:1333-1341.

21. Seeger TF, Bartlett B, Coskran TM, et al. Immunohistochemical localization of PDE10A in the rat brain. Brain Res. 2003;985:113-126. 